\title{
Dynamic Texture Detection, Segmentation and Analysis
}

\author{
B.U.Toreyin, Y.Dedeoglu, \\ A.E.Cetin \\ Bilkent University \\ Ankara, Turkey \\ \{bugur,yigithan,cetin\}@bilkent.edu.tr
}

\author{
S.Fazekas, D.Chetverikov \\ SZTAKI \\ Budapest, Hungary \\ fazekas@vision.sztaki.hu \\ csetverikov@sztaki.hu
}

\author{
T.Amiaz, N.Kiryati \\ Tel Aviv University \\ Israel \\ nk@eng.tau.ac.il
}

\begin{abstract}
Dynamic textures are common in natural scenes. Examples of dynamic textures in video include fire, smoke, clouds, trees in the wind, sky, sea and ocean waves etc. In this showcase, (i) we develop real-time dynamic texture detection methods in video and (ii) present solutions to video object classification based on motion information.
\end{abstract}

\section{Keywords}

Dynamic textures in video, fire detection, smoke detection

\section{OBJECTIVE OF THE SHOWCASE PROJECT}

Researchers extensively studied 2-D textures and related problems in the field of image processing. On the other hand, there is very little research on dynamic texture detection in video. It is well known that tree leaves in the wind, moving clouds etc. cause major problems in outdoor video motion detection systems. If one can initially identify bushes, trees, and clouds in a video, then such regions can be excluded from the search space or proper care can be taken in such regions, and this leads to robust moving object detection and identification systems in outdoor video. One can take advantage of the research in 2-D textures to model the spatial behaviour of a given dynamic texture. To be able to detect and segment dynamic textures in challenging real world applications, differences in dynamics must be also analyzed. Two different approaches will be studied in this showcase. In the first approach, dynamic textures are classified as weak dynamic textures will be analysed with standard optical flow algorithms relying on the brightness constancy assumption. However, selfocclusion, material diffusion, and other physical processes not obeying the brightness constancy assumption make such algorithms inappropriate for strong dynamic textures. An alternative to the brightness constancy assumption, the brightness conservation assumption enables the brightness of an image point to propagate to its neighborhood and thus to model complex brightness changes. A non-regular optical flow calculation based on the brightness conservation assumption provides a better model for strong dynamic textures.

Copyright is held by the author/owner(s).

CIVR'07, July 9-11, 2007, Amsterdam, The Netherlands.

Copyright 2007 ACM 978-1-59593-733-9/07/0007.
In the second approach, prior information about dynamic textures is used for detecting smoke and flames in video. It is experimentally observed that flame flicker process is not a narrow-band activity but it is wide-band activity covering 2 to 15 $\mathrm{Hz}$. Zero-crossings of wavelet coefficients covering the band of 2 to $15 \mathrm{~Hz}$ is an effective feature and Hidden Markov Models (HMM) can be trained to detect temporal characteristics of fire using the wavelet domain data. Similarly, temporal behaviour of tree leaves in the wind or cloud motions will be investigated to achieve robust video understanding systems including content based video retrieval systems.

\section{FIRE DETECTION IN VIDEO}

Conventional point smoke and fire detectors typically detect the presence of certain particles generated by smoke and fire by ionisation or photometry. An important weakness of point detectors is that they are distance limited and fail in open or large spaces. The strength of using video in fire detection is the ability to monitor large and open spaces. Current fire and flame detection algorithms are based on the use of color and motion information in video. In this work, we not only detect fire and flame colored moving regions but also analyze the motion [1,2]. It is wellknown that turbulent flames flicker with a frequency of around 10 Hz. Therefore, fire detection scheme was made more robust by detecting periodic high-frequency behavior in flame colored moving pixels compared to existing fire detection systems described in [2]. However, this approach may also produce false alarms to police cars in tunnels. Our experiments indicate that flame flicker frequency is not constant and it varies in time. Variations of a flame pixel in video is plotted in Fig. 1. In fact, variations in flame pixels can be considered as random events. Therefore, a Markov model based modeling of flame flicker process produces more robust performance compared to frequency domain based methods[3]. Similar arguments are also valid for video based smoke detection $[4,5]$.
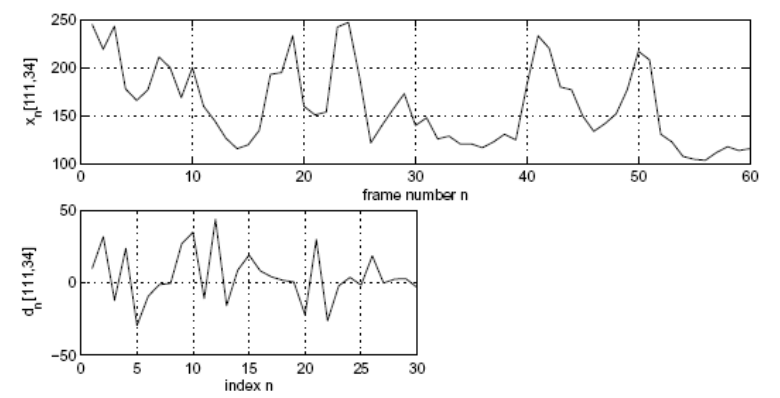

Figure 1. Temporal variation of a flame pixel and the corresponding wavelet coefficients (bottom plot). 
Another important clue for fire detection is the boundary of moving objects in the video. If the contours of an object exhibit rapid time-varying behavior then this is an important sign of presence of flames in the scene. This time-varying behavior is directly observable in the variations of color channel values of the pixels under consideration. Hence, the model is built as consisting of states representing relative locations of the pixels in the color space. When trained with flame pixels off-line, such a model successfully mimics the spatio-temporal characteristics of flames. The same model is also trained with non-flame pixels in order to differentiate between real flames and other flame colored ordinary moving objects.

In addition, there is spatial color variation in flames (cf. Fig.2). Flame pixels exhibit a similar spatial variation in their chrominance or luminosity values, as shown in Fig. 2. The spatial variance of flames are much larger than that of an ordinary flamecolored moving object. The absolute sum of spatial wavelet coefficients of low-high, high-low and high-high subimages of the regions bounded by gray-scale rectangles excerpted from a child's fire colored t-shirt and inside a fire, are shown in Fig. 2 [2]. This feature of flame regions is also exploited by making use of the Markov models. This way of modeling the problem results in less number of false alarms when compared with other proposed methods utilizing only color and ordinary motion information as in [1].

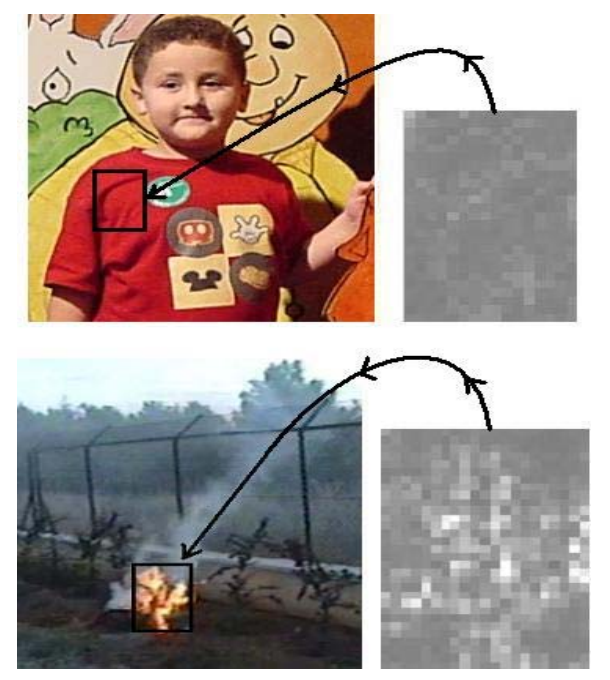

Figure 2. Comparison of spatial variations of fire-colored regions. Flame(bottom-left) have substantially higher spatial variation(bottom-right) compared to an ordinary fire-colored region.

In spatial color analysis step, pixels of flame coloured regions are horizontally and vertically scanned using the same Markov models in temporal analysis. If the fire-coloured model has a higher probability spatially as well, then an alarm is issued.

Our experimental studies indicate that Markovian modeling of the flames is not only more robust than the use of FFT to detect $10 \mathrm{~Hz}$ flame flicker but also computationally more efficient. Details of our experimental studies can be found in [3].

The method can be used for fire detection in movies and video databases as well as real-time detection of fire. It can be incorporated with a surveillance system monitoring an indoor or an outdoor area of interest for early fire detection.

\section{DETECTION OF SWAYING TREE LEAVES}

It is well known that tree leaves and branches in the wind, moving clouds, etc., is a main source of false alarms in outdoor video analysis. If one can initially identify bushes, trees and clouds in a video, then such regions can be excluded from the search space or proper care can be taken in such regions. This leads to robust moving object detection and identification systems in outdoor video. A method for detection of tree branches and leaves in video is developed. It is observed that the motion vectors of tree branches and leaves exhibit random motion. On the other hand regular motion of green colored objects has welldefined directions as shown in Figure 3. In our method, the wavelet transform of motion vectors are computed and objects are classified according to the wavelet coefficients of motion vectors. Color information is also used to reduce the search space in a given image frame of the video. Motion trajectories of moving objects are modeled as Markovian processes and Hidden Markov Models (HMMs) are used to classify the green colored objects in the final step of the algorithm.

Our detection algorithm consists of three main steps: (i) green colored moving region detection in video, (ii) estimation of the motion trajectories and computation of the wavelet domain signals representing motion trajectories, and iii) HMM based classification of the motion trajectories.

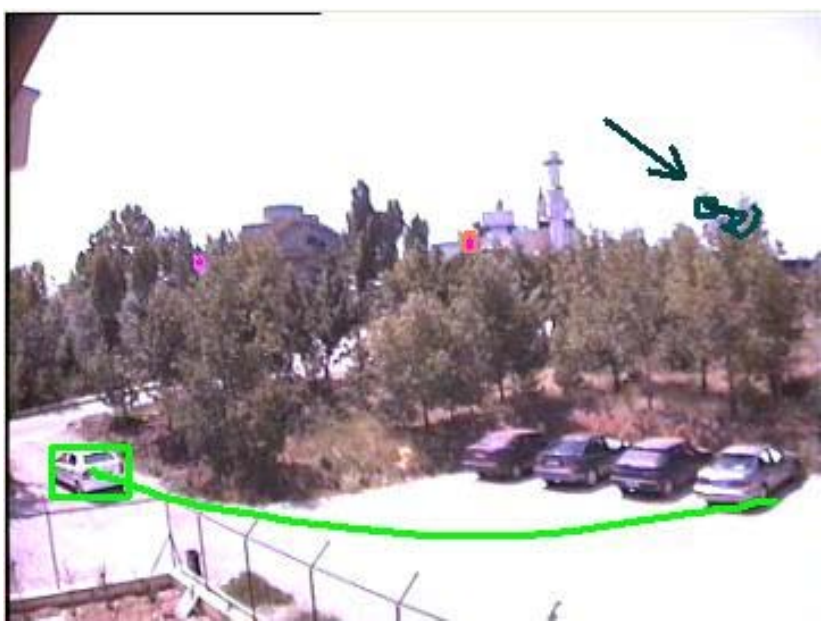

Figure 3. The car has a directionally consistent trajectory whereas the leaves, marked by an arrow, randomly sway in the wind.

A random behavior with low temporal correlation is apparent for leaves and branches of a tree, in both the horizontal and vertical component of the temporal motion signal as shown in Figs. 3, respectively. On the other hand, an ordinary moving object with a well-defined direction does not exhibit such a random behavior. In this case there is high correlation between the samples of the motion feature signal. This difference in motion characteristics is also apparent in the wavelet domain [6]. 


\section{SEGMENTATION AND ANALYSIS OF DYNAMIC TEXTURES}

Dynamic textures are common in natural scenes, however, in many cases only parts of the scene form a dynamic texture. Furthermore, the spatial location and extent of dynamic textures can vary with time and they can be partially transparent. All these make it difficult to separate them from a (moving) textured background. To be able to detect and segment dynamic textures in such challenging cases, differences in dynamics must be analyzed. Color and geometry can be misleading in many cases.

Based on general characteristics of the underlying processes, dynamic textures can be grouped in two main categories: weak and strong dynamic textures. A weak dynamic texture such as a simple moving texture does not posses any intrinsic dynamics. Following it in an appropriate local moving coordinate system i.e. "going with the flow" - a weak dynamic texture becomes static and thus it can be processed with classical techniques. Contrary, characteristics of strong dynamic textures possessing an intrinsic dynamics cannot be fully captured with regular motion compensation.

Weak dynamic textures can be studied with standard optical flow algorithms relying on the brightness constancy assumption. However, self-occlusion, material diffusion, and other physical processes not obeying the brightness constancy assumption make such algorithms inappropriate for strong dynamic textures. An alternative to the brightness constancy assumption, the brightness conservation assumption enables the brightness of an image point to propagate to its neighborhood and thus to model complex brightness changes. A non-regular optical flow calculation based on the brightness conservation assumption models strong dynamic textures more precisely (see Fig. 4). Information encoded in the optical flow can be extracted and analyzed [7].
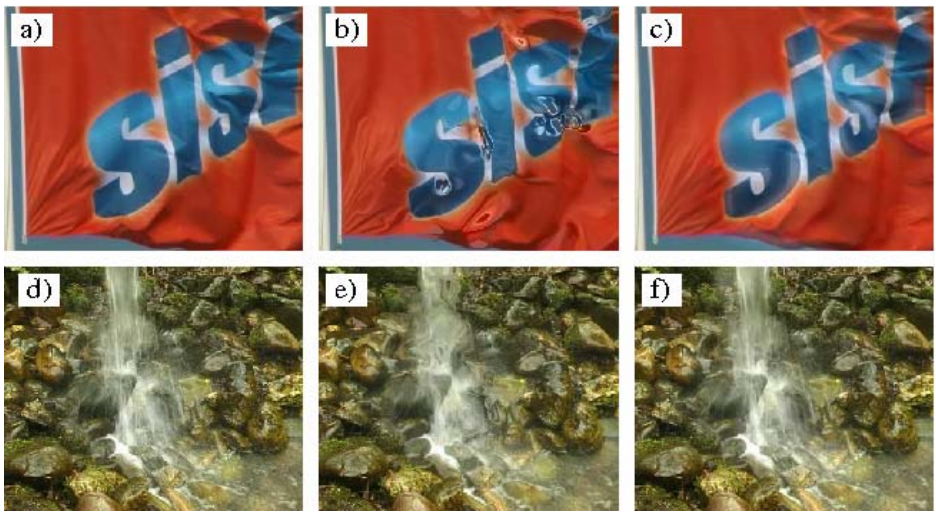

Figure 4. Motion compensation: Original frames (a, d), HornSchunck flow (b, e), non-regular flow (c, f).

While a static or weak dynamic texture obeys brightness constancy, a strong dynamic texture is better modeled with brightness conservation. Using this, we have developed a level set segmentation scheme for identifying and segmenting dynamic texture regions based on their general motion characteristics, without using any color information. We have tested our method on videos of non-segmented dynamic textures showing flowing water, steam, smoke, and fire - all in a natural context [8]. The experimental results show the adequacy of our approach for detecting and segmenting strong dynamic textures in challenging cases [9]. An optimized (less accurate) version of our software works in real-time.
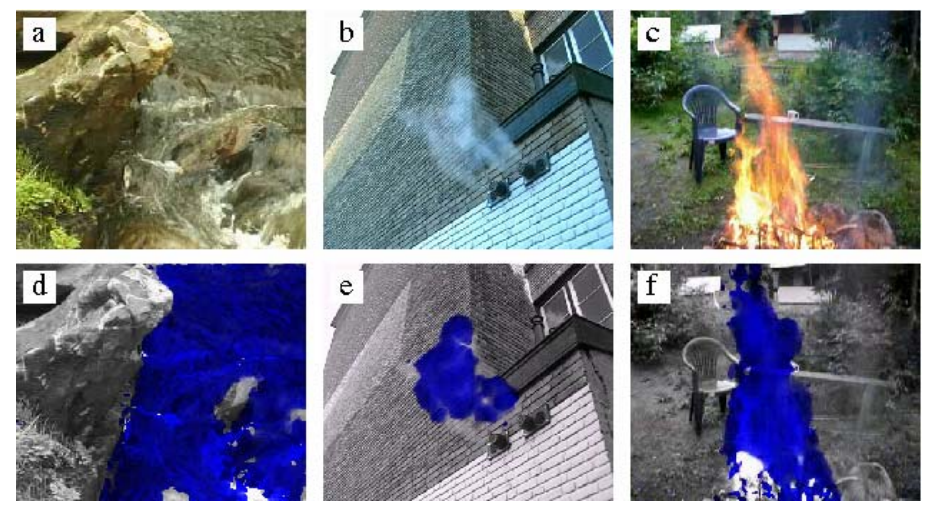

Figure 5. Dynamic texture segmentation: Stream (a, d), Steam (b, e), Fire (c, f).

\section{DEMO AND WEB-SITE}

Sample fire and smoke detection videos and the software can be downloaded from URL:

http://signal.ee.bilkent.edu.tr/VisiFire/index.html

Proposed fire and smoke detection method is also explained at CVonline, compendium of computer vision by Prof.R.B.Fisher of University of Edinburgh at URL:

http://homepages.inf.ed.ac.uk/rbf/CVonline/LOCAL_COPIES/TO REYIN2/index.html

Segmentation of dynamic textures can be reached at:

http://vision.sztaki.hu/ fazekas/dtsegm/CIVR07/

\section{REFERENCES}

[1] Yigithan Dedeoglu, B. Ugur Toreyin, Ugur Gudukbay, A. Enis Cetin, "Real-time Fire and Flame Detection in Video", IEEE 30th Int. Conf. on Acoustics, Speech and Signal Processing, ICASSP’05, Philadelphia, USA, 2005.

[2] B. Ugur Toreyin, Yigithan Dedeoglu, Ugur Gudukbay, A. Enis Cetin, "Computer Vision Based System for Real-time Fire and Flame Detection”, Pattern Recognition Letters, 27 (2006) 49-58. 
[3] B. Ugur Toreyin, Yigithan Dedeoglu, A. Enis Cetin, "Flame detection in video using hidden Markov models”, IEEE Int. Conf. On Image Proc., ICIP 2005, Genoa, Italy.

[4] B. Ugur Toreyin, Yigithan Dedeoglu, A. Enis Cetin, "Wavelet Based Real-Time Smoke Detection in Video", 13th European Signal Processing Conference EUSIPCO 2005, Antalya, Turkey.

[5] B. Ugur Toreyin, Yigithan Dedeoglu, A. Enis Cetin, "Contour Based Smoke Detection in Video Using Wavelets", 14th European Signal Processing Conference EUSIPCO 2006, Florance, Italy.

[6] B. Ugur Toreyin and A. Enis Cetin, "Wavelet Based Detection of Moving Tree Branches and Leaves in Video", IEEE Int. Symposium on Circuits and Systems, ISCAS 2006, Kos, Greece.
[7] S. Fazekas and D. Chetverikov. Normal versus complete flow in dynamic texture recognition: A comparative study. In Int. Workshop on Texture Analysis and Synthesis, pages 3742, 2005.

[8] R. Péteri, M. Huskies, and S. Fazekas. DynTex: A comprehensive database of Dynamic Textures, "http://www.cwi.nl/projects/dyntex", 2006.

[9] T. Amiaz, S. Fazekas, D. Chetverikov, and N. Kiryati. Detecting Regions of Dynamic Texture. In: Int. Conf. on Scale Space and Variational Methods in Computer Vision, 2007 (accepted for publication). 